\title{
Cardiac Computed Tomographic Angiography after Abnormal Ischemia Test as a Gatekeeper to Invasive Coronary Angiography.
}

João Reis ( $\sim$ jpr_911@hotmail.com )

Hospital of Santa Marta: Hospital de Santa Marta https://orcid.org/0000-0003-0047-8306

\section{Ruben Ramos}

Hospital of Santa Marta: Hospital de Santa Marta

Hugo Marques

Hospital of Santa Marta: Hospital de Santa Marta

\section{Pedro Modas Daniel}

Hospital of Santa Marta: Hospital de Santa Marta

\section{Silvia A}

Hospital of Santa Marta: Hospital de Santa Marta

\section{Luis Almeida Morais}

Hospital of Santa Marta: Hospital de Santa Marta

\section{Madalena Cruz}

Hospital of Santa Marta: Hospital de Santa Marta

\section{Rita Moreira}

Hospital of Santa Marta: Hospital de Santa Marta

\section{André Monteiro}

Hospital of Santa Marta: Hospital de Santa Marta

\section{Duarte Cacela}

Hospital of Santa Marta: Hospital de Santa Marta

\section{Luisa Figueiredo}

Hospital of Santa Marta: Hospital de Santa Marta

\section{Rui Cruz Ferreira}

Hospital of Santa Marta: Hospital de Santa Marta

\section{Research Article}

Keywords: Coronary Computed Tomography Angiography, Invasive Coronary Angiography, Myocardial Ischemia Testing, Coronary Artery Disease.

Posted Date: August 4th, 2021 
DOl: https://doi.org/10.21203/rs.3.rs-651078/v1

License: (c) (1) This work is licensed under a Creative Commons Attribution 4.0 International License. Read Full License

Version of Record: A version of this preprint was published at The International Journal of Cardiovascular Imaging on February 28th, 2022. See the published version at https://doi.org/10.1007/s10554-02102426-6. 


\section{Abstract}

Objectives

This study aimed to determine the impact of systematic coronary computed tomographic angiography (CCTA) use following an abnormal non-invasive ischemia test (NIST) on patient selection strategy for invasive coronary angiography (ICA).

Background

In patients with suspected stable coronary artery disease (CAD), NIST use frequently results in suboptimal diagnostic and revascularization yields of ICA.

Methods

This randomized clinical trial, conducted at a single academic tertiary center, selected 220 symptomatic patients with mild-to-moderately abnormal NIST results who were referred for ICA. Patients received either the originally intended ICA $(n=105)$ or CCTA $(n=115)$. The primary endpoint was the diagnostic yield of ICA in each group. Revascularization yield and major adverse cardiovascular events at 12 months were also assessed.

Results

The patients were $69 \pm 9$ years old, $60 \%$ were men, and $31 \%$ had typical angina. Mean pre-test probability of obstructive CAD was $34 \%$. Overall prevalence of obstructive CAD was $37.7 \%$ on the index angiographic procedure. In the CCTA group, ICA was cancelled by referring physicians in 83 patients (72.2\%) after receiving CCTA results. For those undergoing ICA, diagnostic $(84.4 \%$ vs $41.7 \%, p<0.001)$ and revascularization $(71.9 \%$ vs $38.8 \%, p=0.001)$ yields were significantly higher for CCTA-guided ICA than for standard NIST-guided ICA. Mean cumulative radiation exposure was significantly lower in the CCTAguided ICA arm than in the NIST-guided ICA arm (12 \pm 9 vs $16 \pm 10 \mathrm{mSv}$, respectively, $p=0.024)$. There were no significant differences in the primary safety endpoint rates between the strategies $(p=0.439)$.

Conclusions

In patients with suspected CAD and mild-to-moderately abnormal ischemia tests, a diagnostic strategy including CCTA as a gatekeeper is safe and effective and significantly improves diagnostic and revascularization yields of ICA.

\section{Introduction}

With the progressive increase in the population at risk for coronary artery disease (CAD), health systems are under considerable logistical and financial pressures to offer efficient methods of risk stratification, diagnosis, and therapy (1). Current international guidelines recommend direct referrals to invasive 
coronary angiography (ICA) in patients with a high clinical likelihood of CAD and the use of non-invasive testing to select intermediate-risk patients who may need ICA (2). However, this patient selection strategy based on alternative functional or anatomic testing may result in sub-optimal diagnostic performance of ICA (3). Up to $50 \%$ of men and $65 \%$ of women undergoing elective ICA do not have obstructive CAD, usually resulting in no further diagnostic or therapeutic interventions (4), particularly if a functional test is used as the initial diagnostic strategy $(5,6)$. Therefore, designing better diagnostic algorithms is necessary to select patients who are candidates for ICA.

Coronary computed tomographic angiography (CCTA) is an excellent method to non-invasively exclude obstructive CAD and has been included in diagnostic algorithms as an alternative to non-invasive ischemia tests (NIST) $(7,8)$ - Fig. 1. However, its systematic and sequential use as a gatekeeper to ICA in symptomatic patients after abnormal NIST findings is not fully defined.

This study aimed to test the hypothesis that, in non-high-risk patients with suspected stable CAD who are referred for ICA following abnormal NIST results, a strategy that systematically includes the sequential performance of CCTA will increase the diagnostic and revascularization yields of ICA compared to the conventional strategy.

\section{Methods}

\section{Study participants}

The study included patients aged $\geq 18$ years who were referred for ICA based on clinical suspicion of de novo CAD and an abnormal NIST.

Patients with uncontrolled severe angina, acute coronary syndromes, previously known CAD, atrial fibrillation, inability to obtain a steady sinus rhythm, chronic kidney disease or previous kidney transplantation, contrast allergy, or other CCTA-specific contraindications were excluded. Patients with an abnormal NIST classified as severe or reported as inconclusive were also excluded. All other NIST abnormalities were accepted as mild or moderate. Ischemia severity was determined by an NIST reading and/or referring physicians and was not adjudicated by investigators. Detailed patient selection criteria are listed in Table 1.

\section{Study design}

We conducted a prospective, open-label, randomized controlled trial in a single academic tertiary center between January 2015 and December 2018, to compare the diagnostic yield of ICA (detection of obstructive $C A D$ ) from two different patient selection strategies.

Patients were recruited after referral to ICA and were only included in the study if the attending physician consented. The randomization process used a computer-generated sequentially numbered list. Investigators and participants were blinded to the randomization sequence but not to the assigned group. In the conventional ICA strategy, patients were allocated to undergo ICA, as originally intended. In the 
selective strategy, patients were allocated to undergo CCTA, and the results were made available to the patients and referring physician. The final decision on whether to proceed or not with ICA was taken by the attending clinician. Investigators did not intervene in clinical management.

All patients were followed-up by telephone interviews and clinical chart reviews for at least 12 months after undergoing CCTA or index ICA, whichever occurred later.

The investigation conforms to the principles outlined in the Declaration of Helsinki. The institutional ethics committee approved the study protocol. All patients provided written informed consent for participation in the study according to the standards approved by the local ethics committee.

\section{Baseline data and non-invasive ischemia tests}

Baseline data on demographic, clinical, and symptomatic status were recorded, including data on cardiovascular risk scores (Framingham and European SCORE) $(9,10)$. The pre-test probability of obstructive CAD was determined per the Duke clinical score (11).

\section{Cardiac computed tomography angiography, invasive coronary angiography, and revascularization}

CCTA was performed following the selective arm strategy. From January 2015 to May 2016 a 64-detector computed tomography (CT) scanner (VCT 64 Lightspeed, GE healthcare, Wisconsin, USA) was used. Thereafter, the exams were performed on a dual-source 128-detector CT scanner (Somatom Definition Flash, Siemens, Erlangen, Germany). Acquisition protocols, interpretation, and reporting were performed according to local standardized practice. Coronary calcium quantification and classification per the Agatston method were carried out in all cases (12). The investigators classified the CCTA results as "negative" (in the case of normal coronary arteries or nonobstructive CAD) or "positive" (in the presence of obstructive CAD defined as at least one segment with stenosis $\geq 70 \%$, left main stenosis $\geq 50 \%$, high calcium score precluding CT angiogram as determined by the attending imager or by the presence of any uninterpretable segment) (13).

Regardless of the randomization group, when the referring clinician decided to proceed to ICA, it was performed within a period not exceeding 3 months after randomization. The ICA was performed per the current institutional standards by operators who were not involved in the study. Obstructive CAD was defined as stenosis in $\geq 50 \%$ of the left main coronary artery or stenosis in $\geq 70 \%$ in any coronary vessel with at least $1.5 \mathrm{~mm}$ diameter. A normal coronary artery was defined as the complete absence of any luminal narrowing detectable by angiography. Nonobstructive CAD was defined as CAD not meeting the criteria for obstructive CAD or normal coronary arteries.

After undergoing diagnostic ICA, the decision to proceed with revascularization or not (percutaneous or surgical) for each patient was taken by the attending cardiologist and interventional operator and was not conditioned by any protocol specification of the study.

\section{Primary endpoint}


The primary endpoint of the study was the ICA diagnostic yield, which was defined as the proportion of patients with obstructive coronary disease in each arm as determined by ICA.

\section{Secondary endpoints}

1. Rates of ICA in the patients randomized to the group of selective ICA. To determine if a stratification strategy that included CCTA applied to a population with stable CAD and abnormal NIST could decrease referrals for ICA. This pre-specified secondary endpoint was reached if the referral rate for ICA in the selective group was at least $40 \%$ lower than that in the control group.

2. Revascularization yield (percutaneous or surgical). To determine whether the use of CCTA in the selective strategy ultimately led to higher rates of revascularization (proportion of revascularized patients with respect to the total number of patients undergoing ICA).

\section{Any major adverse cardiovascular events $\mathbf{1 2}$ months after randomization. A combined endpoint that} includes all-cause death, non-fatal myocardial infarction, unstable angina (angina recurring or requiring hospitalization, urgent revascularization), non-fatal stroke, or cardiovascular hospitalization.

4. Mean cumulative radiation exposure in each group. We assessed the overall radiation exposure (in millisieverts) using the dose-length product (converted using factors of $0.014 \mathrm{mSv} / \mathrm{mGy} \times \mathrm{cm}$ ) for CT and modeled radiation and published survey data for ICA, percutaneous coronary intervention, and myocardial perfusion imaging (7 mSv, $15 \mathrm{mSv}$, and $14 \mathrm{mSv}$, respectively) (14).

5. Mean cumulative contrast dose in each group. Contrast used in CCTA, ICA, and percutaneous coronary intervention, when performed, was included.

\section{Statistical analysis}

The study tested the following null hypothesis $\left(\mathrm{H}_{0}\right), \pi 1$ (primary endpoints in the selective strategy) $=\pi 2$ (primary endpoints in the direct strategy), and an alternative hypothesis $\left(H_{1}\right), \pi 1$ (primary endpoint in the selective strategy) $\neq \pi 2$ (primary endpoints in the direct strategy), using the bilateral $\chi 2$ test for a significance level of 0.05 , power of 0.8 , and a risk of occurrence of the first primary endpoint (obstructive CAD on ICA) of $50 \%$ in the direct ICA strategy, and $80 \%$ on the selective ICA. This estimate is based on the expected prevalence of obstructive CAD of $50 \%$ (15) and negative predictive value of CCTA $>90 \%$ described in the literature (16). We estimated that a sample of 120 individuals in each study arm would be needed to verify a reduction in the normalcy rate of ICA from $50-20 \%$, with $95 \%$ confidence, and $80 \%$ potency.

Continuous data were assessed using the unpaired t-test or Mann-Whitney test, and categorical data were assessed by $\chi 2$ or Fisher tests, as appropriate. Normally distributed continuous variables are expressed as mean $\pm S D$, and non-normally distributed data are expressed as medians and interquartile ranges. All data were analyzed using SPSS 20.0 (IBM, NY, USA).

\section{Results}


Of the 2256 patients referred for ICA during the study period, 236 patients were enrolled after application of exclusion criteria. Additionally, 5 patients were excluded due to the withdrawal of written consents and one due to non-cardiovascular death before randomization. A total of 231 patients (10.2\%) were included, of which 115 patients were randomly allocated to the CCTA-selective strategy group and 116 patients to the conventional strategy group. In the latter group, 11 patients were excluded after withdrawing consent or declining to undergo ICA. Twelve-month follow-up data were available for all patients who underwent the conventional ICA strategy. Six patients were lost to follow-up after CCTA in the selective ICA arm; of these, one had obstructive CAD on CCTA (Fig. 2).

Detailed baseline population characteristics are described in Table 2. The mean age of the study cohort was $68.7 \pm 9.3$ years, and $59.5 \%$ were men. Almost one-third of the study population (31.8\%) had persistent typical angina, and $95.4 \%$ were under an anti-anginal medication.

Except for a significantly higher prevalence of diabetes $(37.1 \%$ vs $24.3 \%, p=0.039)$ in the conventional arm, the demographic and clinical profiles of the two groups were well-balanced. The burden of angina, traditional clinical risk scores, and the pre-test likelihood of CAD (34.6\% vs $33.2 \%, p=0.586)$ were also similar between the study arms, as was the proportion of patients who underwent single-photon emission computed tomography (SPECT) scans and exercise treadmill stress tests. Differences in post-test Duke's Treadmill Scores were non-significant $(-1.1 \pm 8.7$ vs $-1.4 \pm 4.5, p=0.740)$

The prevalence of obstructive CAD in the overall study population in the index procedure (as defined either by CCTA or ICA) was $37.7 \%$ (Fig. 3). Although the prevalence of any CAD was higher in the CCTA group ( $68.7 \%$ vs $55.2 \%)$, there were no statistically significant differences in the prevalence of obstructive CAD $(41.9 \%$ vs $33.9 \%, p=0.222)$. Coronary anatomy and findings of each index procedure are described in Table 3. Thirteen patients referred for CCTA did not undergo coronary angiograms as a result of extensive coronary calcification, which could render some coronary segments uninterpretable. As per the study protocol definition, these patients were classified as having obstructive CAD (13).

The primary endpoint in the CCTA-guided arm was $84.4 \%$ (27 of 32 referred patients had obstructive CAD on ICA), which was significantly higher than that observed in the conventional ICA arm $(41.9 \%, 44$ of 105 referred patients, $p<0.001$, Fig. 4). In the selective ICA strategy, ICA was cancelled in 83 of the patients (72.2\%) after receiving CCTA results, thereby significantly reducing the number of patients who were referred for ICA and meeting one pre-specified secondary endpoint of the study-the reduction of the ICA referral rate by at least $40 \%$. Around $45 \%$ of these patients did not have CAD, while $47 \%$ presented nonobstructive CAD. Seven patients with obstructive CAD documented by CCTA were not referred for ICA because they were considered to have low-risk coronary anatomy or they became asymptomatic after optimization of their anti-anginal therapy, or both.

The revascularization yield of ICA was also significantly higher in the selective strategy than in the conventional ICA strategy $(75.0 \%$ vs $40.0 \%, p=0.002$; Fig. 4). Surgical revascularization was performed in $6.9 \%$ patients included in the selective arm and in $11.4 \%$ patients in the conventional arm $(p=0.249$,

Table 4).

Page $7 / 18$ 
Overall, the mean cumulative radiation exposure was significantly superior in the conventional ICA arm $(15.5 \pm 10.4 \mathrm{mSv}$ vs $12.3 \pm 9.0 \mathrm{mSv}, p=0.024)$. For the 32 patients in the selective strategy undergoing both CCTA and ICA, the mean radiation dose was similar to that for the patients in the conventional arm (15.4 mSv vs $15.5 \mathrm{mSv}, p=0.994$ ). If subsequent percutaneous revascularization was excluded, the difference in the cumulative radiation dose between the conventional group and the selective strategy arm was not significant ( $11.5 \pm 4.2 \mathrm{mSv}$ vs $9.9 \pm 3.3 \mathrm{mSv}, p=0.108)$. Similarly, there were no overall differences in the mean cumulative contrast doses in each group ( $90.8 \mathrm{~mL}$ vs $86.3 \mathrm{~mL}, p=0.588$ ). For the 32 patients in the selective strategy group undergoing both CCTA and ICA, the mean contrast dose was similar to that of the patients in the conventional arm (93.9 mL vs $90.8 \mathrm{~mL}, p=0.870$; Fig. 5).

Overall, $2.8 \%$ of patients in the study population experienced major adverse cardiovascular events (MACEs) during the first 12-month follow-up; however, no deaths were reported (Table 5). During followup, $1.7 \%(n=2)$ of patients in the selective referral arm and 3.8\% $(n=4)$ in the direct referral arm experienced MACEs $(p=0.439)$. There were no significant differences in the individual MACE endpoints between the strategies, and none of these events were related to the ICA. Additionally, at 12 months, the conventional ICA strategy was not significantly different from the CCTA-based selective strategy with respect to the burden of persistent angina $(24.8 \%$ vs $29.4 \%, p=0.450)$.

\section{Discussion}

In this study, we observed that the systematic use of CCTA as a gatekeeper in this population reduced the number of patients inappropriately referred for ICA by $72 \%$. For those who ultimately underwent ICA, the diagnostic and revascularization yields were significantly improved in the selective referral arm. This was achieved without compromising safety since the 12-month MACE rates, cumulative radiation exposures, and administered contrast volumes were similar.

Previous studies have either directly compared the diagnostic or prognostic performances of CCTA versus NIST at early stages in the diagnostic cascade or allowed for inclusion of a large majority of patients with no previous positive NIST in pragmatic trials assessing CCTA gatekeeping merits (17-20). Therefore, although CCTA has established diagnostic and prognostic roles in the management of stable CAD as an alternative to NIST, its role as a systematic anatomic gatekeeper in patients referred for ICA after positive NIST results remains incompletely understood. To our knowledge, this is the first randomized trial to study the performance of the systematic use of CCTA in a specific population of patients with a positive NIST referred for ICA.

A cohort of patients who were candidates for ICA from a large observational registry (PLATFORM study) were included for CCTA combined with a non-invasive CT-derived fractional flow reserve as a gatekeeper strategy (21). In line with our results, this resulted in an ICA cancellation rate of $61 \%$ and a marked reduction in the finding of no obstructive CAD at ICA. However, this study did not report on CCTA performance without coupled functional imaging. 
In the recently published ISCHEMIA trial, most patients with moderate-to-severe ischemia underwent CCTA before randomization to exclude left main disease or nonobstructive CAD. This strategy resulted in the exclusion of $14 \%$ of patients from the trial due to non-invasive findings of no obstructive CAD (22). These findings highlight the importance of non-invasive anatomical assessment before undergoing ICA even in a moderate-to-high risk cohort.

Concurrent with other studies $(23,24)$, this trial revealed that CCTA-guided ICA showed significantly higher revascularization yields than the conventional strategy because patients with no obstructive CAD were eliminated from undergoing ICA. However, the overall revascularization rate was significantly lower in the selective arm (24/115 patients) than in the direct ICA arm (40/105 patients). In addition to the confident exclusion of obstructive CAD due to its high negative predictive value, CCTA may also allow for further ICA cancellation after the coronary anatomy is known, even if obstructive CAD is found to be present. In our study, of the 39 patients with evidence of obstructive CAD on CCTA in the selective arm, ICA was subsequently cancelled by attending physicians in 7 patients (18\%). This was due to clinicians preferring to defer ICA in cases where prognostic or symptomatic benefits from revascularization were not expected from the observed low-risk obstructive coronary anatomy.

In this study, enrollment occurred after patients were already referred for ICA by treating physicians. The low-to-intermediate pre-test probability of obstructive CAD could argue against the decision to refer patients at this risk level to undergo ICA. However, indications for referral were in accordance with societal guidelines, were considered appropriate, and largely reflected the high rates of normal ICA observed in real-world routine practice.

The limitations of this study must be acknowledged. The actual prevalence of obstructive CAD observed in this study (38\%) was lower than the expected (50\%), based on our institution's previous registry. This may have amplified the benefits of the CCTA-selective ICA strategy. Thus, the study findings may not be generalizable to populations where obstructive CAD prevalence is generally higher. Despite the exclusion criteria in the study, a relatively high proportion of patients (11\%) referred for CCTA did not undergo coronary angiograms because of extensive coronary calcification. This may be a potential limitation of the proposed diagnostic approach. In the future, a CT-derived ischemic index may have an important role in this patient subset.

Our results may not be reproducible if non-invasive functional imaging other than exercise electrocardiogram and SPECT is used. However, the accuracy of imaging NIST is highly dependent on local expertise, and the reported accuracies largely overlap under comparable conditions. Further, although no significant differences were observed between the arms with respect to safety events at one year, at a low level of event rate, the study was not powered to draw firm conclusions on these secondary endpoints. Given the recent results of the ISCHEMIA trial, it is unlikely that alternative diagnostic strategies in a cohort of patients with a low-to-moderate ischemia would drive major prognostic differences. This study was conducted before the ISCHEMIA trial; thus, the conventional ICA referral strategy after NIST may be influenced, in the future, by the neutral results of the trial. Moreover, the pivotal 
role of CCTA in excluding nonobstructive CAD and left main coronary artery disease (5\% of the study population) further supports a selective CCTA-guided diagnostic strategy.

In conclusion, in patients with suspected CAD and mild-to-moderately abnormal ischemia test results, in whom ICA is considered, a diagnostic strategy that includes the systematic use of CCTA as a gatekeeper may safely reduce the number of unnecessary invasive procedures, leading to a significant improvement in the diagnostic and revascularization yields of ICA. These results should be further validated in independent cohorts of CAD patients before implementing a screening approach in clinical practice.

\section{Abbreviations}

$\mathrm{CAD}=$ coronary artery disease; $\mathrm{CCTA}=$ coronary computed tomographic angiography; NIST = noninvasive ischemia test; ICA = invasive coronary angiography; CCS = Canadian Cardiovascular Society; CT = computed tomography; $\mathrm{MACE}=$ major adverse cardiovascular event

\section{Declarations}

Funding: No grant or funding was received for this study.

Conflict of interest: All authors declare that they have no conflicts of interest.

Relationship with industry: All authors declare that they have no relationship with industry.

\section{References}

1. European Heart Network. European cardiovascular disease statistics 2017. Available at: htttp://www.ehnheart.org/cvd-statistics/cvd-statistics-2017.html. Accessed January 31, 2020.

2. Knuuti J, Wijns W, Saraste A, Saraste A, Capodanno D, Barbato E, Funck-Brentano C, Prescott E, Storey RF, Deaton C, Cuisset T, Agewall S, Dickstein K, Edvardsen T, Escaned J, Gersh BJ, Svitil P, Gilard M, Hasdai D, Hatala R, Mahfoud F, Masip J, Muneretto C, Valgimigli M, Achenbach S, Bax JJ. ESC Scientific Document Group. 2019 ESC Guidelines for the diagnosis and management of chronic coronary syndromes. Eur Heart J 2020; 41:407-77. https://dx.doi.org/10.1093/eurheartj/ehz425.

3. Patel MR, Peterson ED, Dai D, Brennan JM, Redberg RF, Anderson HV, Brindis RG, Douglas PS. Low diagnostic yield of elective coronary angiography. N Engl J Med 2010; 362:886-95. https://dx.doi.org/10.1056/NEJMoa0907272.

4. Patel MR, Dai D, Hernandez AF, Douglas PS, Messenger J, Garratt KN, Maddox TM, Peterson ED, Roe MT. Prevalence and predictors of nonobstructive coronary artery disease identified with coronary angiography in contemporary clinical practice. Am Heart J 2014; 167:846-52.e2. https://dx.doi.org/10.1016/j.ahj.2014.03.001.

5. Neglia D, Rovai D, Caseli C, Pietila M, Teresinska A, Aguadé-Bruix S, Pizzi MN, Todiere G, Gimelli A, Schroeder S, Drosch T, Poddighe R, Casolo G, Anagnostopoulos C, Pugliese F, Rouzet F, Le Guludec D, 
Cappelli F, Valente S, Gensini GF, Zawaideh C, Capitanio S, Sambuceti G, Marsico F, Perrone Filardi P, Fernández-Golfín C, Rincón LM, Graner FP, de Graaf MA, Fiechter M, Stehli J, Gaemperli O, Reyes E, Nkomo S, Mäki M, Lorenzoni V, Turchetti G, Carpeggiani C, Marinelli M, Puzzuoli S, Mangione M, Marcheschi P, Mariani F, Giannessi D, Nekolla S, Lombardi M, Sicari R, Scholte AJ, Zamorano JL, Kaufmann PA, Underwood SR, Knuuti J; EVINCI Study Investigators. Detection of significant coronary artery disease by noninvasive anatomical and functional imaging. Circ Cardiovasc Imaging 2015; 8:e002179. Doi:10.1161/CIRCIMAGING.114.002179.

6. Douglas P, Hoffmann U, Patel M, Mark DB, Al-Khalidi HR, Cavanaugh B, Cole J, Dolor RJ, Fordyce CB, Huang M, Khan MA, Kosinski AS, Krucoff MW, Malhotra V, Picard MH, Udelson JE, Velazquez EJ, Yow E, Cooper LS, Lee KL; PROMISE Investigators. Outcomes of anatomical versus functional testing for coronary artery disease. N Engl J Med 2015; 372:1291-300.

https://dx.doi.org/10.1056/NEJMoa1415516.

7. Lubbers M, Dedic A, Coenen A, Galema T, Akkerhuis J, Bruning T, Krenning B, Musters P, Ouhlous M, Liem A, Niezen A, Hunink M, de Feijter P, Nieman K. Calcium imaging and selective computed tomography angiography in comparison to functional testing for suspected coronary artery disease: the multicentre, randomized CRESCENT trial. Eur Heart J 2016; 37:1232-43. https://dx.doi.org/10.1093/eurheartj/ehv700.

8. Williams MC, Hunter A, Shah ASV, Assi V, Lewis S, Smith J, Berry C, Boon NA, Clark E, Flather M, Forbes J, McLean S, Roditi G, van Beek EJR, Timmis AD, Newby DE; SCOT-HEART Investigators. Use of coronary computed tomographic angiography to guide management of patients with coronary disease. J Am Coll Cardiol 2016; 67:1759-68. https://dx.doi.org/10.1016/j.jacc.2016.02.026.

9. D'Agostino RB Sr, Vasan RS, Pencina MJ, Wolf PA, Cobain M, Massaro JM, Kannel WB. General cardiovascular risk profile for use in primary care: the Framingham Heart Study. General cardiovascular risk profile for use in primary care: the Framingham Heart Study. Circulation 2008; 117:743-53. https://dx.doi.org/10.1161/CIRCULATIONAHA.107.699579.

10. Conroy RM, Pyörälä K, Fitzgerald AP, Sans $S$, Menotti A, De Backer $G$, De Bacquer $D$, Ducimetière $P$, Jousilahti P, Keil U, Njølstad I, Oganov RG, Thomsen T, Tunstall-Pedoe H, Tverdal A, Wedel H, Whincup P, Wilhelmsen L, Graham IM; SCORE project group. Estimation of ten-year risk of fatal cardiovascular disease in Europe: the SCORE project. Eur Heart J 2003; 24:987-1003.

https://dx.doi.org/10.1016/s0195-668x(03)00114-3.

11. Mark DB, Hlatky MA, Harrell FE Jr, Lee KL, Califf RM, Pryor DB. Exercise treadmill score for predicting prognosis in coronary artery disease. Ann Intern Med 1987; 106:793-800.

https://dx.doi.org/10.7326/0003-4819-106-6-793.

12. Agatston AS, Janowitz WR, Hildner FJ, Zusmer NR, Viamonte M Jr, Detrano R. Quantification of coronary artery calcium using ultrafast computed tomography. J Am Coll Cardiol 1990; 15:827-32. https://dx.doi.org/10.1016/0735-1097(90)90282-t.

13. Park MJ, Jung JI, Choi YS, Ann SH, Youn HJ, Jeon GN, Choi HC. Coronary CT angiography in patients with high calcium score: evaluation of plaque characteristics and diagnostic accuracy. Int $\mathrm{J}$ Cardiovasc Imaging 2011; 27 Suppl 1:43-51. https://dx.doi.org/10.1007/s10554-011-9970-7. 
14. Gerber TC, Carr JJ, Arai AE, Dixon RL, Ferrari VA, Gomes AS, Heller GV, McCollough CH, McNitt-Gray MF, Mettler FA, Mieres JH, Morin RL, Yester MV. lonizing radiation in cardiac imaging: a science advisory from the American Heart Association Committee on Cardiac Imaging of the Council on Clinical Cardiology and Committee on Cardiovascular Imaging and Intervention of the Council on Cardiovascular Radiology and Intervention. Circulation 2009; 119:1056-65. https://dx.doi.org/10.1161/CIRCULATIONAHA.108.191650.

15. Rio P, Ramos R, Pereira-da-Silva T, Barbosa C, Cacela D, Fiarresga A, de Sousa L, Abreu A, Patrício L, Bernardes L, Ferreira RC. Yield of contemporary clinical strategies to detect patients with obstructive coronary artery disease. Heart Int 2016; 10:e12-9. https://dx.doi.org/10.5301/heartint.5000224.

16. Meijboom WB, Meijs MF, Schuijf JD, Cramer MJ, Mollet NR, van Mieghem CA, Nieman K, van Werkhoven JM, Pundziute G, Weustink AC, de Vos AM, Pugliese F, Rensing B, Jukema JW, Bax JJ, Prokop M, Doevendans PA, Hunink MG, Krestin GP, de Feyter PJ. Diagnostic accuracy of 64-slice computed tomography coronary angiography: a prospective, multicenter, multivendor study. J Am Coll Cardiol 2008; 52:2135-44. https://dx.doi.org/10.1016/j.jacc.2008.08.058.

17. SCOT-HEART investigators. CT coronary angiography in patients with suspected angina due to coronary heart disease (SCOT-HEART): an open-label, parallel-group, multicentre trial. Lancet 2015; 385:2383-91. https://dx.doi.org/10.1016/S0140-6736(15)60291-4.

18. Douglas PS, Hoffmann U, Patel MR, Mark DB, Al-Khalidi HR, Cavanaugh B, Cole J, Dolor RJ, Fordyce $\mathrm{CB}$, Huang M, Khan MA, Kosinski AS, Krucoff MW, Malhotra V, Picard MH, Udelson JE, Velazquez EJ, Yow E, Cooper LS, Lee KL; PROMISE Investigators. Outcomes of anatomical versus functional testing for coronary artery disease. N Engl J Med 2015; 372:1291-300. https://dx.doi.org/10.1056/NEJMoa1415516.

19. Dewey M, Rief M, Martus P, Kendziora B, Feger S, Dreger H, Priem S, Knebel F, Böhm M, Schlattmann P, Hamm B, Schönenberger E, Laule M, Zimmermann E. Evaluation of computed tomography in patients with atypical angina or chest pain clinically referred for invasive coronary angiography: randomised controlled trial. BMJ 2016; 355:i5441. https://dx.doi.org/10.1136/bmj.i5441.

20. Chang HJ, Lin FY, Gebow D, An HY, Andreini D, Bathina R, Baggiano A, Beltrama V, Cerci R, Choi EY, Choi JH, Choi SY, Chung N, Cole J, Doh JH, Ha SJ, Her AY, Kepka C, Kim JY, Kim JW, Kim SW, Kim W, Pontone G, Valeti U, Villines TC, Lu Y, Kumar A, Cho I, Danad I, Han D, Heo R, Lee SE, Lee JH, Park HB, Sung JM, Leflang D, Zullo J, Shaw LJ, Min JK Selective referral using CCTA versus direct referral for individuals referred to invasive coronary angiography for suspected CAD: a randomized, controlled, open-label trial. J Am Coll Cardiol Img 2019; 12:1303-12. https://dx.doi.org/10.1016/j.jcmg.2018.09.018.

21. Douglas PS, De Bruyne B, Pontone G, Patel MR, Norgaard BL, Byrne RA, Curzen N, Purcell I, Gutberlet M, Rioufol G, Hink U, Schuchlenz HW, Feuchtner G, Gilard M, Andreini D, Jensen JM, Hadamitzky M, Chiswell K, Cyr D, Wilk A, Wang F, Rogers C, Hlatky MA; PLATFORM Investigators. 1-year outcomes of FFRCT-guided care in patients with suspected coronary disease: the PLATFORM study. J Am Coll Cardiol. 2016; 68:435-45. https://dx.doi.org/10.1016/j.jacc.2016.05.057. 
22. Maron DJ, Hochman JS, Reynolds HR, Bangalore S, O'Brien SM, Boden WE, Chaitman BR, Senior R, López-Sendón J, Alexander KP, Lopes RD, Shaw LJ, Berger JS, Newman JD, Sidhu MS, Goodman SG, Ruzyllo W, Gosselin G, Maggioni AP, White HD, Bhargava B, Min JK, Mancini GBJ, Berman DS, Picard MH, Kwong RY, Ali ZA, Mark DB, Spertus JA, Krishnan MN, Elghamaz A, Moorthy N, Hueb WA, Demkow M, Mavromatis K, Bockeria O, Peteiro J, Miller TD, Szwed H, Doerr R, Keltai M, Selvanayagam JB, Steg PG, Held C, Kohsaka S, Mavromichalis S, Kirby R, Jeffries NO, Harrell FE Jr, Rockhold FW, Broderick S, Ferguson TB Jr, Williams DO, Harrington RA, Stone GW, Rosenberg Y; ISCHEMIA Research Group. Initial invasive or conservative strategy for stable coronary disease. N Engl J Med 2020; 382:1395-407. https://dx.doi.org/10.1056/NEJMoa1915922.

23. Lucas FL, Siewers AE, Malenka DJ, Wennberg DE. Diagnostic-therapeutic cascade revisited: coronary angiography, coronary artery bypass graft surgery, and percutaneous coronary intervention in the modern era. Circulation. 2008; 118:2797-802. https://dx.doi.org/10.1161/CIRCULATIONAHA.108.789446.

24. Newby DE. CONSERVE your energy and resources. J Am Coll Cardiol Img 2019; 12:1313-5. https://dx.doi.org/10.1016/j.jcmg.2018.10.009.

\section{Tables}

Tables 1-5 are not available with this version

\section{Figures}




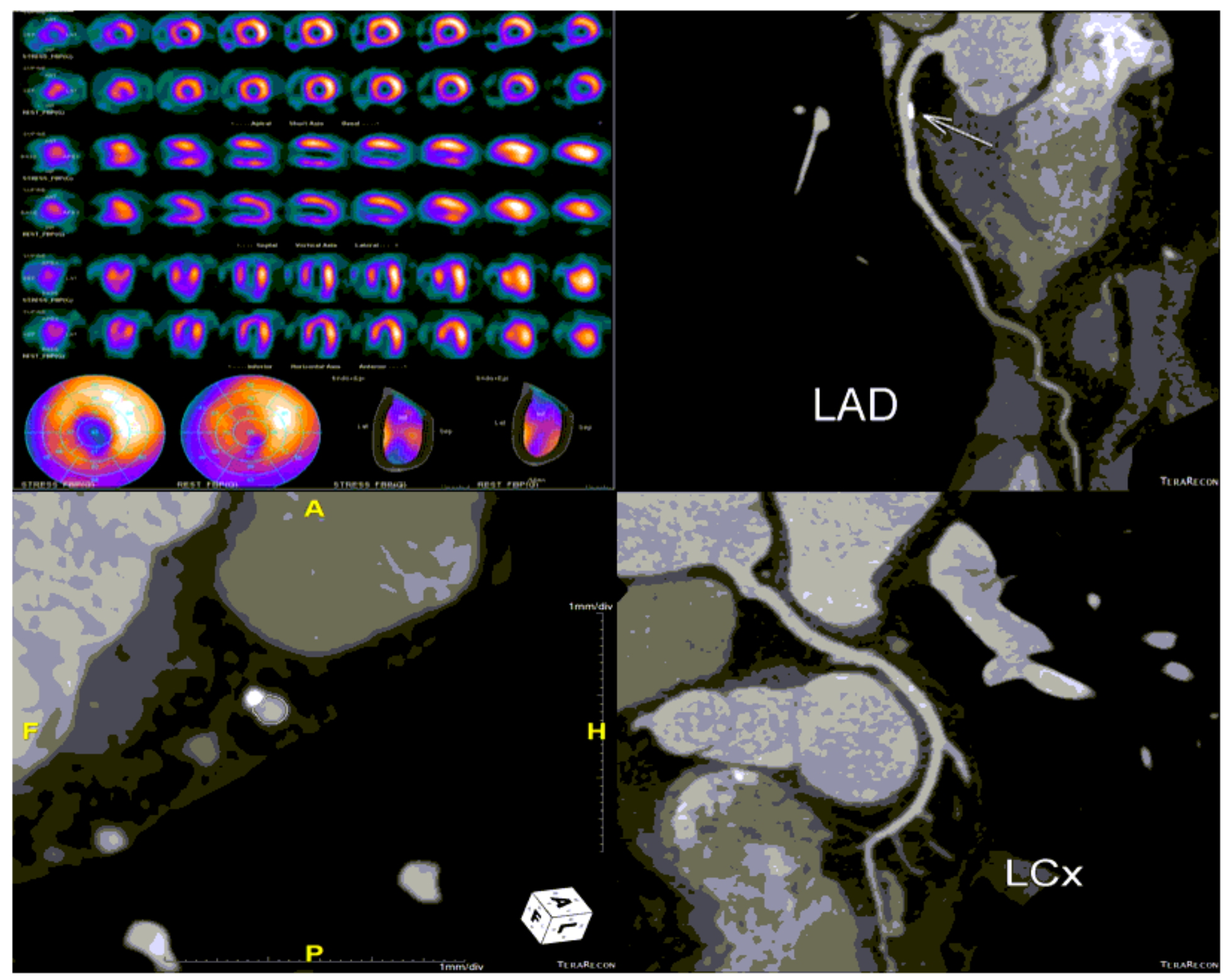

\section{Figure 1}

(A) Cardiac single-photon emission computerized tomography (SPECT) revealing anterior ischemia. (B), (C) CCTA showing a non-obstructive plaque in the proximal left anterior descending artery. (D) Circumflex artery with no coronary artery disease in a patient with left dominant coronary circulation. 


\section{Stable symptomatic patients already referred to ICA}

for diagnosis of CAD based on positive NIST

$(n=1285)$

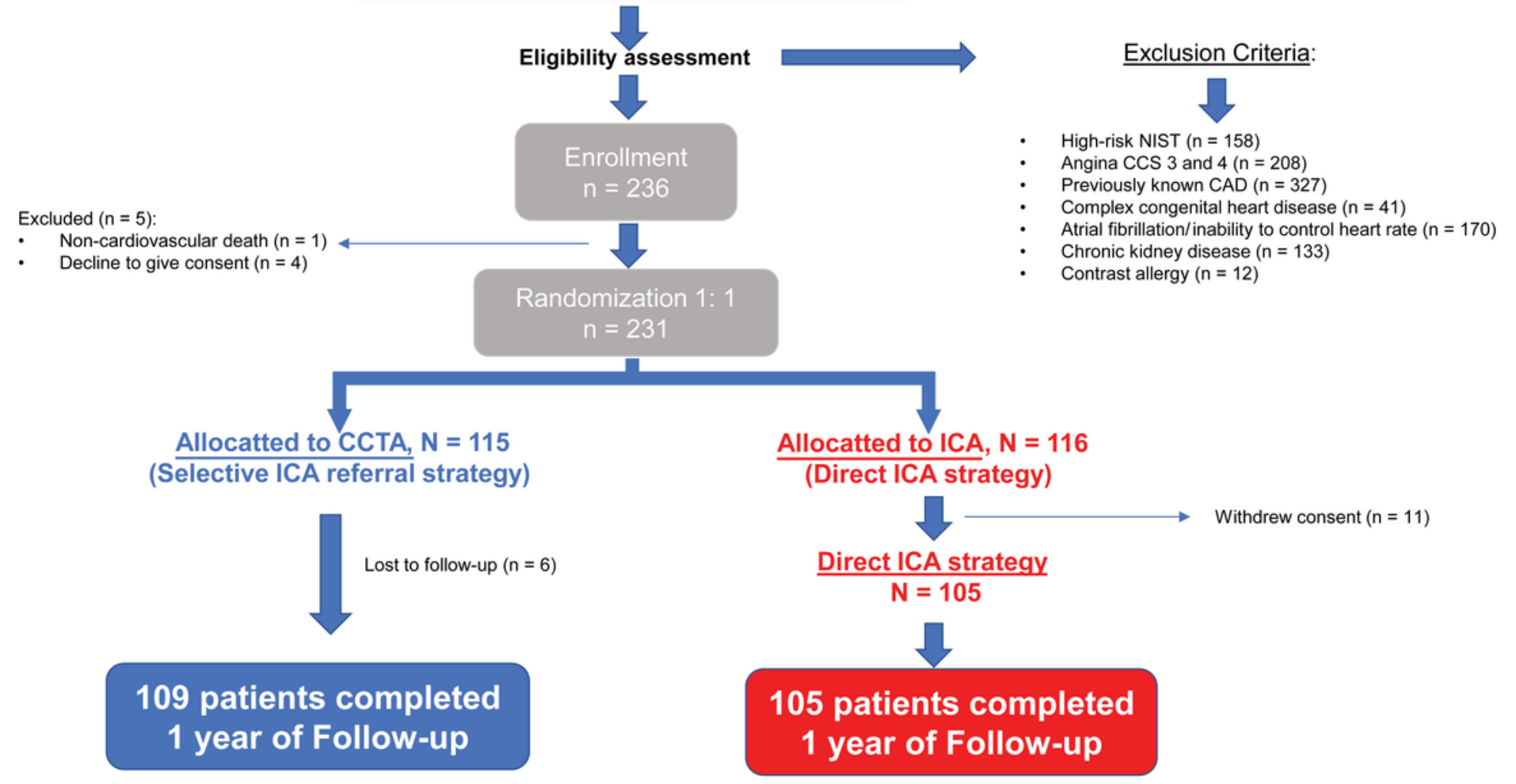

\section{Figure 2}

Study workflow: Non-high-risk patients were randomized for direct invasive coronary angiography (ICA) or coronary computed tomography angiography (CCTA). In the selective coronary angiography group, CCTA results were disclosed and subsequent referral for ICA was left to the discretion of the clinician. 


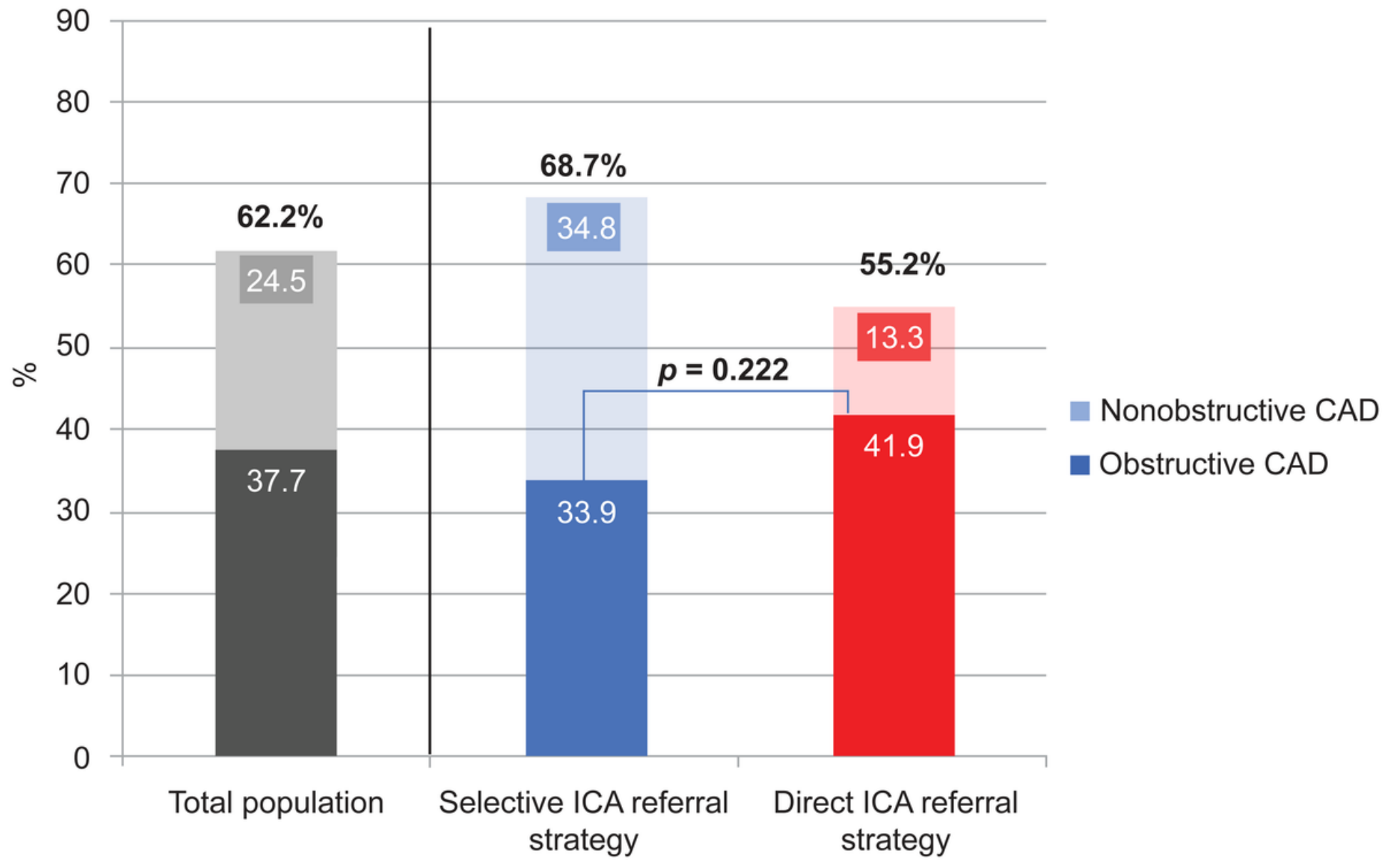

Figure 3

Prevalence of coronary artery disease (CAD) on the index procedure in each strategy. CAD was more frequently detected in the coronary computed tomography angiography (CCTA)-guided arm than in the conventional arm. However, this difference was mainly driven by the rate of nonobstructive CAD. 


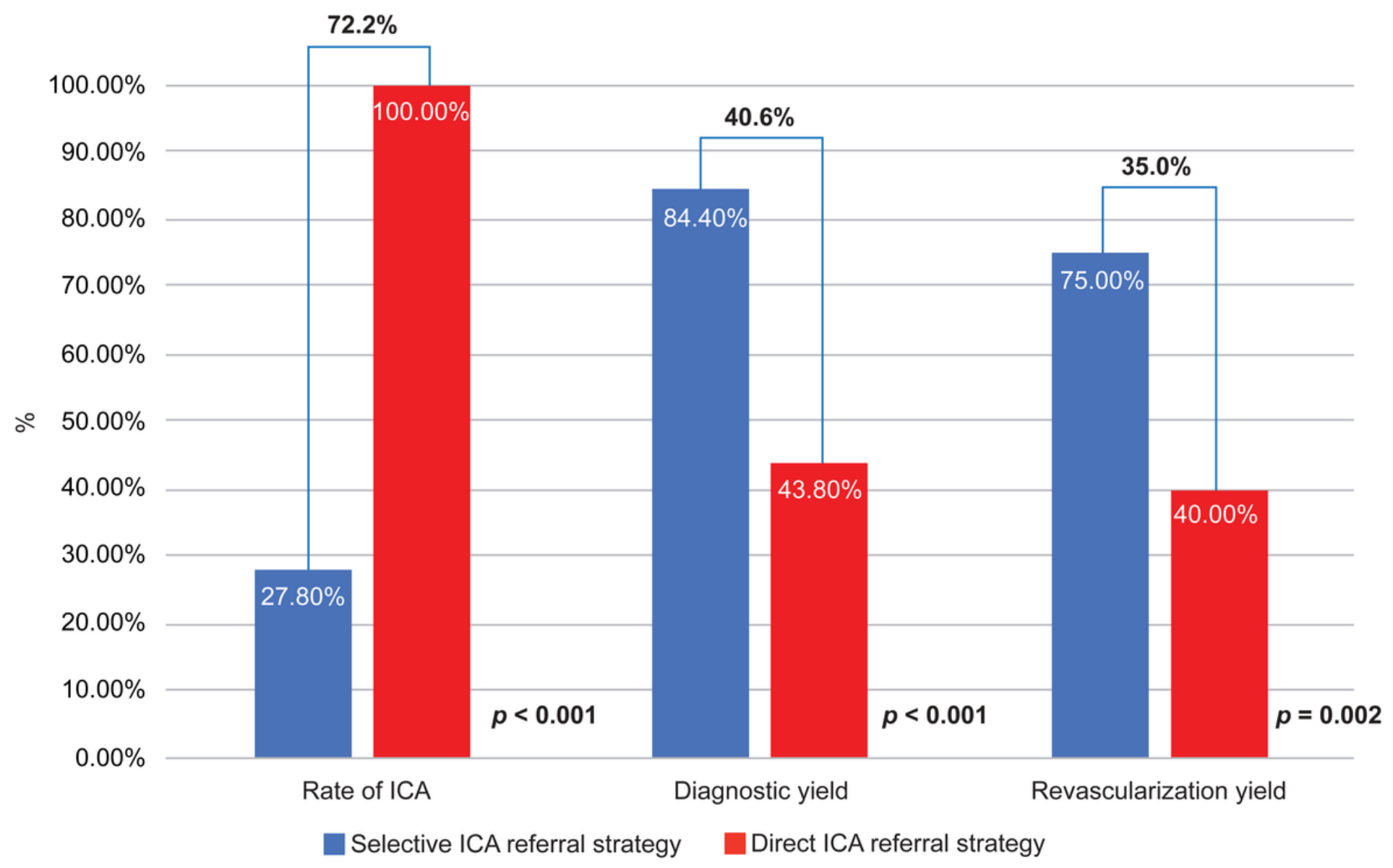

\section{Figure 4}

Central Illustration Rates, diagnostic yields, and revascularization yields of invasive coronary angiography in both groups are shown. Less than a third of patients randomized to the selective coronary computed tomography angiography (CCTA)-guided strategy ultimately underwent invasive coronary angiography (ICA). When ICA was performed, both the diagnostic yield and revascularization yield were significantly higher in the CCTA-guided arm than in the conventional arm. 


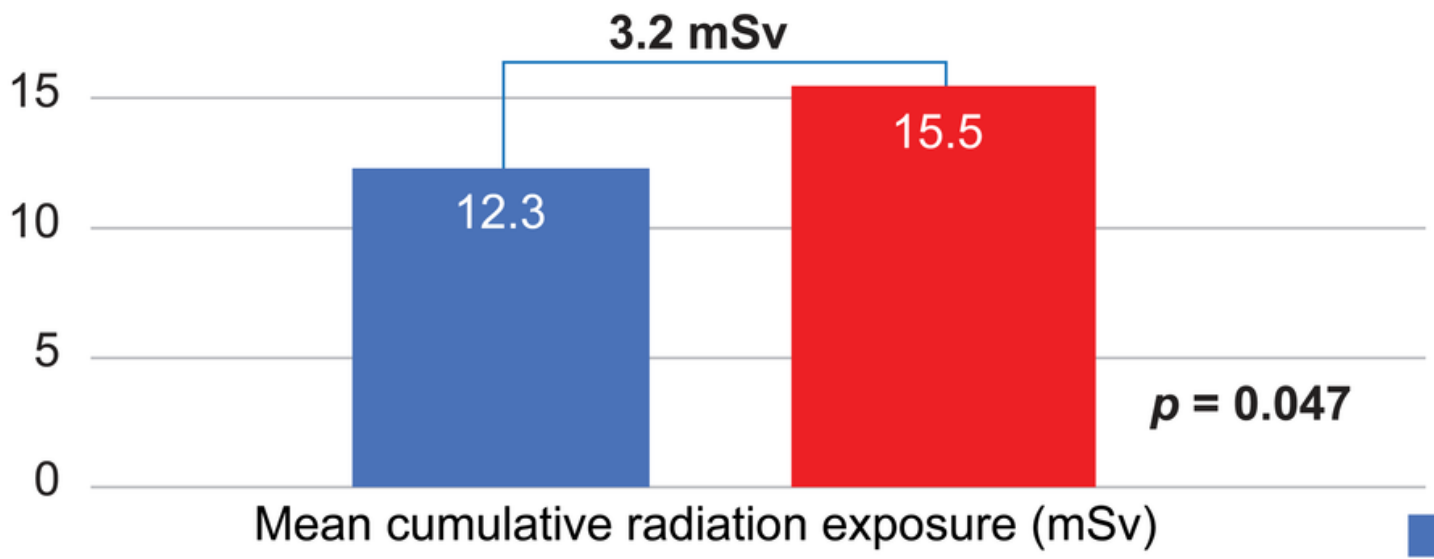

Selective ICA referral strategy

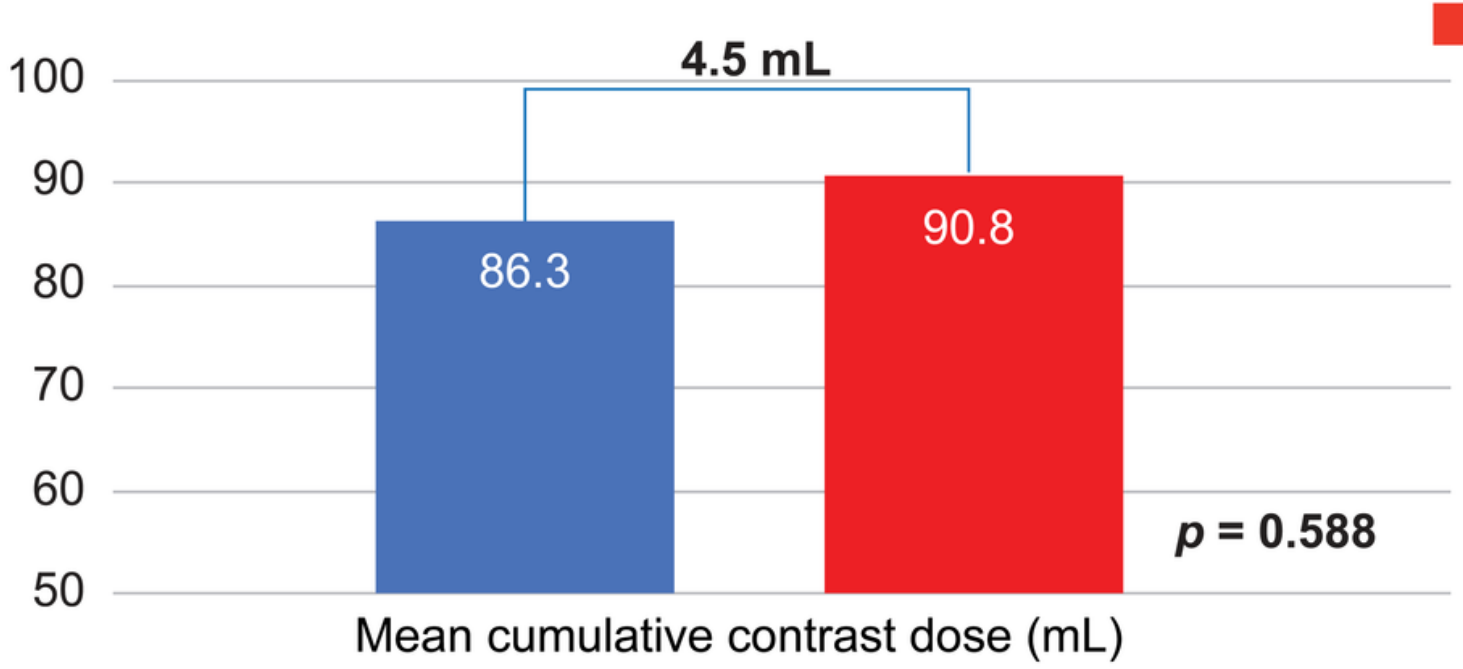

Direct ICA strategy

Figure 5

Mean cumulative radiation and contrast exposure in each group. 